\title{
Coupling of split ring resonators in a mu-negative volumetric metamaterial
}

\author{
J. Machac, J. Zehentner, M. Blaha \\ Czech Technical University in Prague, Technicka 2, 16627 Prague, Czech Republic
}

\begin{abstract}
This paper presents an experimental and theoretical study of the coupling of split ring resonators, and aims to show ways to widen the frequency band of the response of mu-negative metamaterials. The behaviour of these metamaterials is theoretically described in terms of coupled resonant circuits theory. In particular the coupling is studied on pairs of split ring resonators and systems containing the greater number of resonators. Widening of the metamaterial response frequency band is caused primarily by dispersion of the resonant frequencies of the particular resonators, and to a minor extent by mutual coupling. The more particles participate in the tested system, the wider the frequency band of the system response. The requirement to obtain an isotropic medium is taken into account.

Index Terms - Split ring resonator, coupled resonators, mu negative metamaterial, isotropic metamaterial, widening frequency band.
\end{abstract}

\section{INTRODUCTION}

Volumetric mu-negative (MNG) metamaterials, i.e., metamaterials with negative effective permeability and positive permittivity consisting of anisotropic resonant particles - split ring resonators (SRR) - are studied in this paper. There is only a narrow frequency band in which the effective permeability of the metamaterial is negative, due to the resonant behavior of SRR, and this seriously limits their technical applications.

To obtain a volumetric and possibly isotropic MNG metamaterial, SRRs must be properly distributed in space. There are two basic kinds of such metamaterials. Basic cells in the form of a cube with SRRs on its faces, with the tetrahedral symmetry, periodically located in the space, create an isotropic metamaterial [1]. On the other hand, SRRs alone can be randomly distributed in space to obtain an isotropic metamaterial $[1,2]$. We used planar broadside coupled split ring resonators (BC-SRR) [2], which consist of two split rings located on the two sides of the substrate, as shown in Fig. 1.

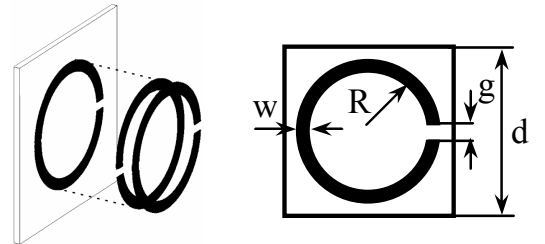

Fig. 1 Planar particle used in MNG metamaterials - broad side coupled SRR.

The resonators, distributed in a proper way in the space, are coupled together in various manners. This coupling widens the frequency band of the metamaterial as a direct analogy of coupled resonators. The other, and more important, effect taking place in systems of a large number of particles and leading to band widening is the dispersion of the geometrical and/or material parameters of the particles. The behaviour of coupled BC-SRRs is studied both at the CST Microwave Studio, and experimentally. The mutual coupling of identical particles is investigated separately from couples of particles detuned due their different parameters. Next, the paper presents the behaviour of the quaternion of the resonators, and finally there is an investigation of real metamaterials, consisting of systems of particles.

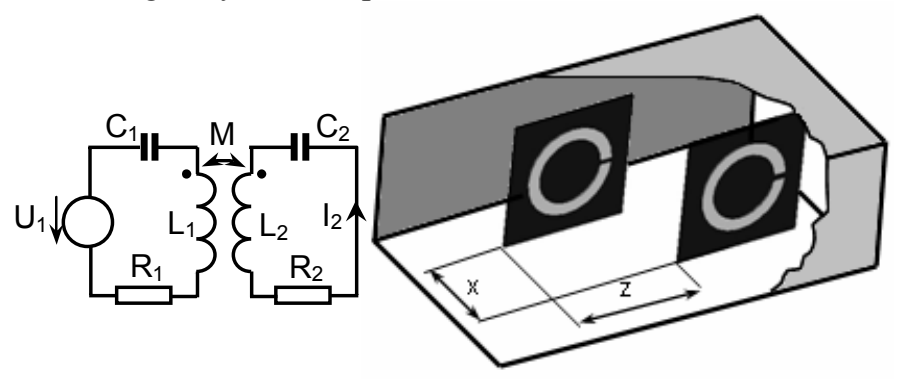

(a)

(b)

Fig. 2 Coupled resonant circuits, equivalent circuit (a), two coupled $\mathrm{BC}-\mathrm{SRR}$ in a waveguide (b).

\section{A COUPLE OF RESONANT CIRCUITS, COUPLED SRRS}

The overall transmission of a couple of resonators depends on the coupling coefficient $k$. The transmission admittance of the circuit in Fig. 2a can be found elsewhere, e.g., in [3]. The frequency band of the response of the circuit from Fig. 2a can by widened in two ways. Fig. 3 shows the transmission admittance modulus as a function of frequency in dependence on the coupling coefficient and keeping all parameters constant, $R_{1}=R_{2}=0.1 \Omega, L_{1}=L_{2}=1 \mathrm{nH}, C_{1}=C_{2}=2.6 \mathrm{pF}$, chosen to get the response around $3.1 \mathrm{GHz}$, similar to the resonant frequency of the investigated particles. The change of the coupling coefficient can be achieved simply by changing the mutual position of particular inductors/particles in space. A similar effect can be obtained by keeping the coupling coefficient constant and varying other circuit parameters. This is an analogy to the couple of particles with different parameters, and thus with different resonant frequencies.

The TEM waveguide with a rectangular cross-section 20x10 mm was used for the numerical simulation at the CST Microwave Studio. The BC-SRR that were used have the same dimensions: side-width $d=7 \mathrm{~mm}$ of the squared 
substrate, $0.127 \mathrm{~mm}$ in thickness and permittivity 2.2 , ring strip-width $w=0.7 \mathrm{~mm}$ and inner radius of the ring $R=1.8$ $\mathrm{mm}$. The fabricated particles do not have equal resonant frequencies due to the dispersion of their dimensions caused by the tolerances of a non-perfect fabrication process and even due to non-homogeneity of the substrate thickness and its permittivity. This dispersion of the resonant frequencies was taken into account in the Microwave Studio model by assuming different widths of the gap $g$, Fig. 1, which directly determines the SRR resonant frequency. The response of the TEM waveguide with a single BC-SRR for the two different values of the ring split-slot $g=0.2 \mathrm{~mm}$ and $g=0.3 \mathrm{~mm}$ calculated by the CST Microwave Studio is shown in Fig. 4. The particle is located in the middle of the waveguide parallel to the side walls. The resonant frequency is $f_{r 1}=3.024 \mathrm{GHz}$ for $g=0.2 \mathrm{~mm}$ and $f_{r 2}=3.056 \mathrm{GHz}$ for $\mathrm{g}=0.3 \mathrm{~mm}$. Fig. 4 also shows the two sets of measured transmissions of an R32 waveguide with a single BC-SRR, labeled "1" and " 2 ", used in subsequent experiments.

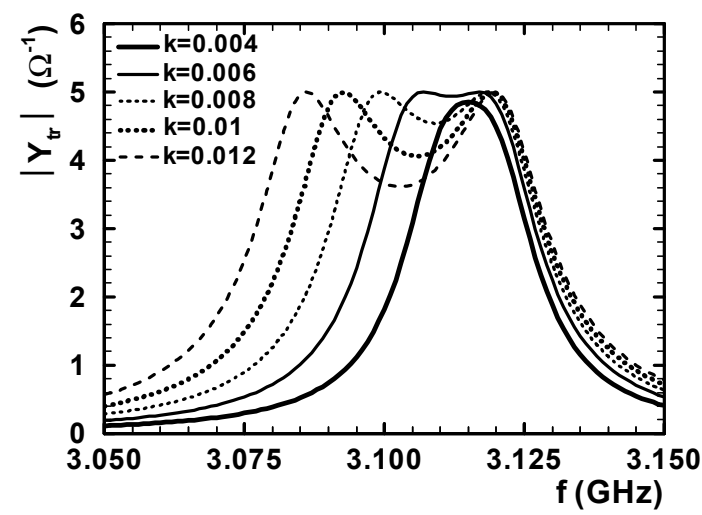

Fig. 3 Calculated transmission admittance of the coupled circuits from Fig. 2a depending on the coupling coefficient [3].

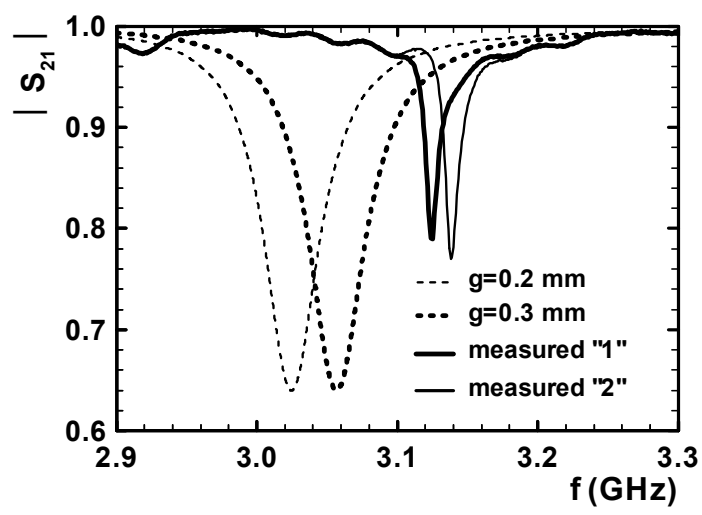

Fig. 4 Calculated transmission of the TEM waveguide with a single BC-SRR with different gap widths and measured transmissions of an R32 waveguide with single BC-SRRs.

When locating the two particles in the waveguide "side-byside", i.e., for $z=0$, Fig. $2 \mathrm{~b}$, their response is different from the response of the coupled circuits. In this case the propagating wave does not see the particles as placed in a cascade, but they create a single element, the response of which is detuned by the mutual inductance and resembles the response of one particle. For the two particles of the same resonant frequency, i.e., of the same gap $g=0.2 \mathrm{~mm}$, this is shown in Table 1. The shape of the transmission characteristic of the TEM waveguide with the couple of these particles is not significantly changed by their position, and looks like the transmission characteristic of a single particle. The tighter the coupling, the higher is the mutual inductance and, consequently, the lower is the resonant frequency. Such behaviour, but of two different BC-SRRs, is documented in Fig. 5 by the results of simulation and verified experimentally. The results are plotted in Fig. 6. We measured similar data for a couple of resonators with equal resonant frequency.

\begin{tabular}{|c|c|}
\hline $\begin{array}{c}\text { distance } x \\
(\mathrm{~mm})\end{array}$ & $\begin{array}{c}\text { resonant frequency } \\
(\mathrm{GHz})\end{array}$ \\
\hline 1 & 2.599 \\
\hline 3 & 2.942 \\
\hline 5 & 3.018 \\
\hline 7 & 3.024 \\
\hline
\end{tabular}

Table 1 Calculated resonant frequency of the couple of identical BCSRRs from Fig.2b in dependence on their distance $x$ calculated for $z$ $=0$.

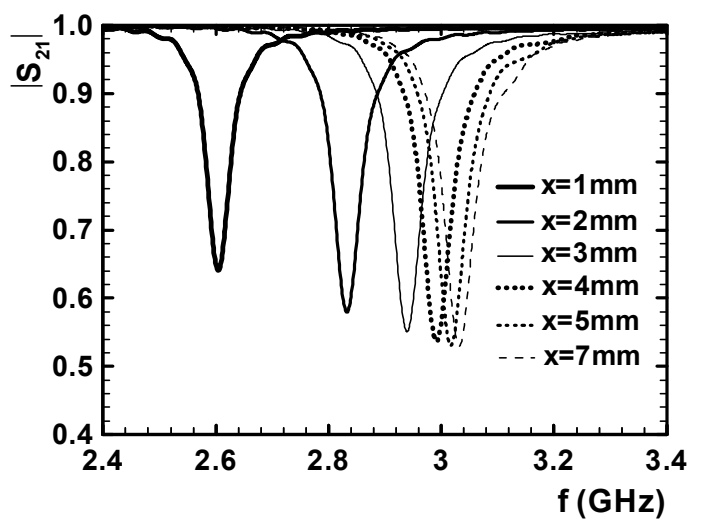

Fig. 5 Calculated transmission of the TEM waveguide with two BC-SRRs with different gaps 0.2 and $0.3 \mathrm{~mm}$ in dependence on their position defined in Fig. $2 \mathrm{~b}$ for $z=0 \mathrm{~mm}$, i.e., side-by-side position.

The two particles located in the waveguide in a cascade, i.e., for nonzero value of $z$ defined in Fig. 2b, do not exactly behave as coupled resonant lumped circuits. Varying their position, we change the shape of the transmission characteristic, but not like in Fig. 3. The transmission characteristics of the couple of two identical BC-SRRs, calculated by the Microwave Studio in the TEM waveguide or measured in an R32 waveguide, show only very weak dependence of their shape on the positions of the particles. The response is detuned by the mutual inductance, as shown in Figs. 5 and 6. The calculated transmission characteristics of the two BC-SRRs detuned by different gaps 0.2 and $0.3 \mathrm{~mm}$ is plotted in Fig. 7 in dependence on their position. For small 
distances $z$ the frequency response is slightly wider than a single particle response, shown in Fig. 4. At $z \geq 5 \mathrm{~mm}$ the coupling is weak and the final characteristic is just the superposition of the particular characteristics. This, however, results in a sufficiently wider response than a single particle provides. The very similar response of real BC-SRRs measured in an R32 waveguide is presented in Fig. 8.

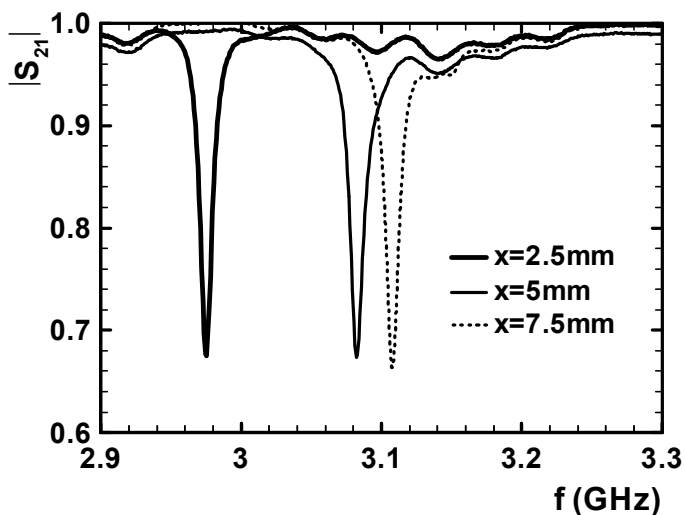

Fig. 6 Measured transmission of an R32 waveguide with two BCSRRs detuned by $12.3 \mathrm{MHz}$ in dependence on their position defined in Fig. $2 \mathrm{~b}$ for $z=0 \mathrm{~mm}$, side-by-side position.

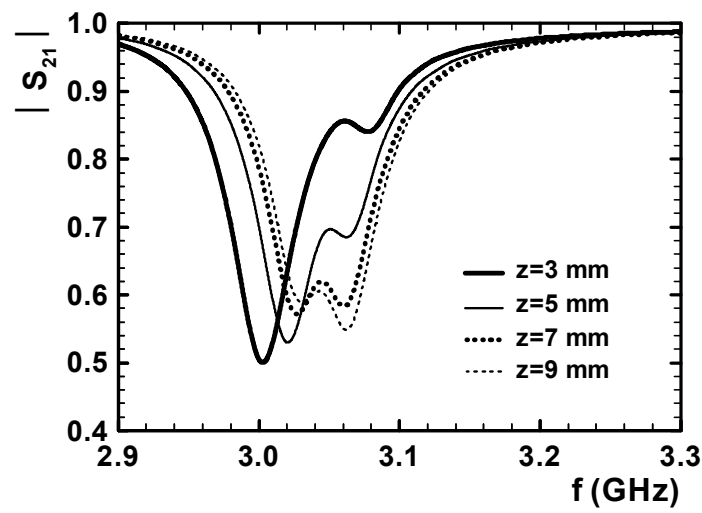

Fig. 7 Calculated transmission of the TEM waveguide with two BCSRRs with different gaps 0.2 and $0.3 \mathrm{~mm}$ in dependence on their position defined in Fig. $2 \mathrm{~b}$ for $x=5 \mathrm{~mm}$.

\section{SYSTEM OF PARTICLES - VOLUMETRIC METAMATERIAL}

The real volumetric mu-negative metamaterial is composed of a system of a large numbers of BC-SRRs either periodically or randomly distributed in the space [2]. The particles in such a system are coupled to each other with various coupling coefficients determined by their positions. Moreover, the fabricated particles do not have equal resonant frequencies. Consequently the frequency band of the system of particles is wider than that of a single particle. Generally, the more particles are present in the system, the wider the band and the higher the rank of isotropy.

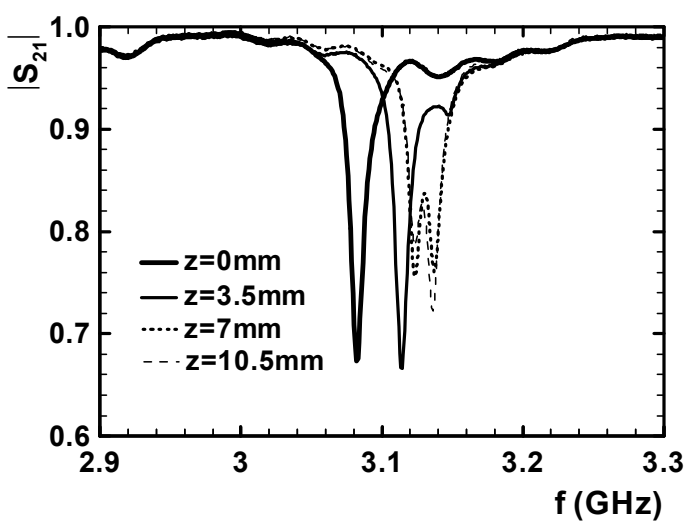

Fig. 8 Measured transmission of an R32 waveguide with two BCSRRs detuned by $12.3 \mathrm{MHz}$ in dependence on their position defined in Fig. $2 \mathrm{~b}$ and for $x=5 \mathrm{~mm}$.

The couples of BC-SRRs were studied in the previous paragraph. Now let us proceed a step toward the real metamaterial composed of the great number of BC-SRRs. Fig. 9 shows the behaviour of four BC-SRRs tuned to different frequencies located in an R32 waveguide according the sketch placed in the plot. The transversal distance of the particles is 5 $\mathrm{mm}$, and the longitudinal distance is $7 \mathrm{~mm}$. The particular measured resonator transmission characteristics are plotted for comparison with the measured characteristic of the quaternion of the resonators. The resulting transmission characteristic is clearly the characteristic of the cascade of four particular particles.

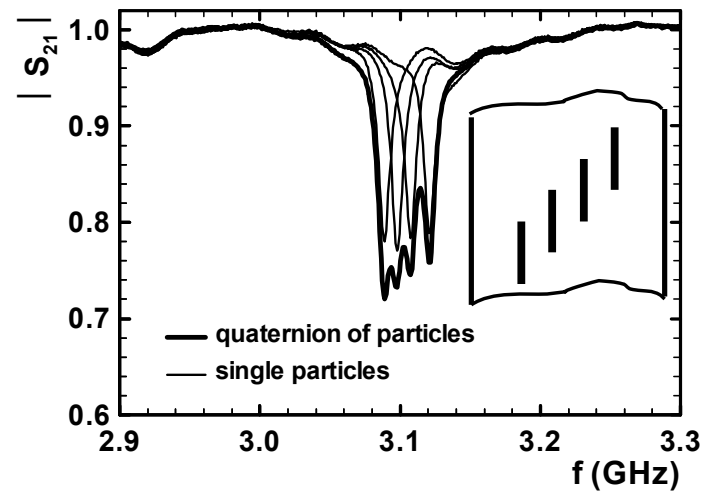

Fig. 9 Measured transmission of an R32 waveguide with 4 BC-SRRs, and of particular resonators.

Finally, we verified and evaluated the effects mentioned above for several different composites. The first tested system consisted of a periodic distribution of particles oriented in the same direction. The response of this medium remained anisotropic, as it was for a single particle. Transmission through this system of 147 epsilon negative particles located in an R32 waveguide, see the inset in Fig. 10, is shown in Fig. 10. The BC-SRRs are located in slots in three polystyrene slices $10 \mathrm{~mm}$ in thickness in rows spaced $4 \mathrm{~mm}$ apart. The frequency band of this medium is considerably wider than that 
of a single particle, as shown in Fig. 4. At the same time, this system of particles has a more intensive response than that of a single particle.

As mentioned above, a very important requirement in many metamaterial applications is the isotropy of their response. As stated, the system shown in Fig. 10 behaves anisotropically, i.e., the response depends on the orientation of the field vectors. However, the isotropic response was obtained using quasi-randomly [1,2] or fully randomly [2] distributed particles. The system consisting of 264 BC-SRRs put into plastic shells in the form of a sphere, see inset of Fig. 11, filling the cube with side length $72 \mathrm{~mm}$ inserted into a raised R32 waveguide exhibits the transmission plotted in Fig. 11. Now the resonators are fully randomly distributed and oriented, so on an average their mutual coupling is less intensive than in the preceding cases. The response of this system is therefore less intensive and its frequency band is narrower than in the preceding case, though still much wider than for a single particle.

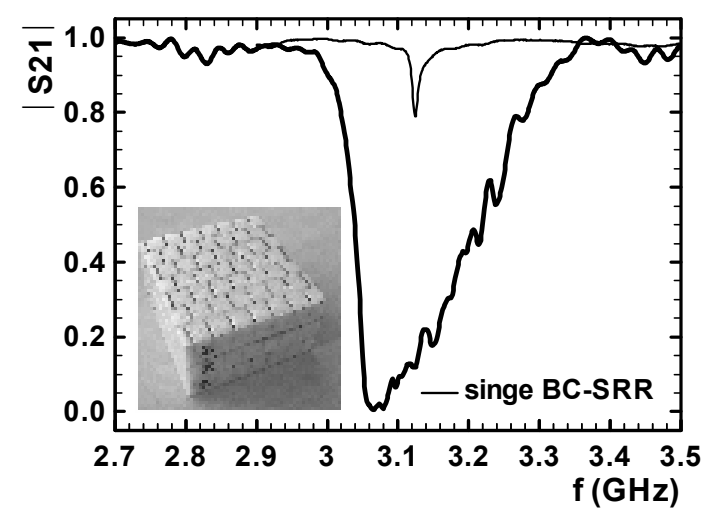

Fig. 10 Measured transmission of an R32 waveguide with 147 BCSRRs periodically distributed and aligned in one direction [1], in comparison with one BC-SRR, Fig. 4.

\section{CONCLUSIONS}

This paper presents how the mutual coupling of BC-SRRs influences their behaviour. The transmission of a couple of BC-SRRs located in the TEM waveguide was calculated by the CST Microwave Studio and measured when located in an R32 waveguide. In spite of these different conditions, the results show similar behaviour. When located side-by-side, the BC-SRRs behave as a single particle with the response similar to the response of a single one. However, the resonant frequency is shifted down due to the detuning caused by the mutual inductance, but with no substantial influence on the shape of the transmission characteristic. Positioning the particles in a cascade widens their response frequency band. This is, however, namely contributed by the dispersion of particle parameters, resulting in the dispersion of their resonant frequencies. Consequently, the more particles compose the metamaterial, the wider is the frequency band of its response. This was proved experimentally on sets of BCSRRs arranged in different ways in an R32 waveguide.

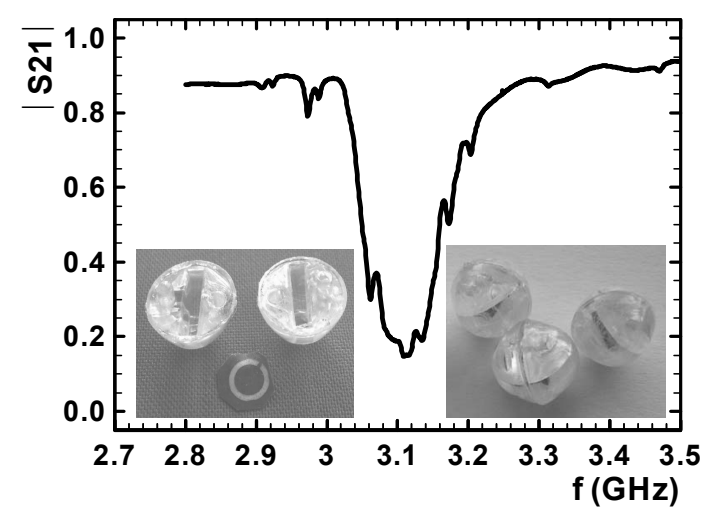

Fig. 11 Measured transmission a squared R32 waveguide with 246 BC-SRRs, when the resonators in the shells are randomly distributed $[2]$.

It turned out that the width of the frequency response of a single BC-SRR in an R32 waveguide is about $30 \mathrm{MHz}$, i.e., $0.97 \%$ of the resonant frequency. Using the system of 147 BC-SRRs periodically distributed and aligned in one direction we obtained the response-width $220 \mathrm{MHz}$, i.e., $7.1 \%$ relative band, defined at the level $-3 \mathrm{~dB}$ of the $\mathrm{S}_{21}$ modulus. The 2D isotropic system consisting of 147 particles periodically distributed but randomly oriented offered a width of $200 \mathrm{MHz}$, i.e., $6.45 \%$. The same system consisting of 243 particles offered a width of $330 \mathrm{MHz}$, i.e., $10.65 \%$. With fully random distribution of the particles, we obtained a response-width of $150 \mathrm{MHz}$, i.e., $4.84 \%$ relative band, for a system consisting of 264 BC-SRRs boxed in plastic spherical shells. This is narrower than that for the $2 \mathrm{D}$ isotropic system, though we now had more particles. This is due to the less intensive mutual coupling between randomly distributed particles than the coupling between particles distributed quasi-periodically.

\section{ACKNOWLEDGEMENT}

This work has been supported by the Grant Agency of the Czech Republic under project 102/06/1106 "Metamaterials, nanostructures and their applications".

\section{REFERENCES}

[1] L. Jelinek, J. Zehentner, J. Machac, "Isotropic magnetic Metamaterials Composed of Periodically and Randomly Arranged Resonating Elements," 2006 Young Scientist Meeting on Metamaterials, Seville, Spain, pp. 54-70.

[2] L. Jelinek, J. Machac, J. Zehentner, "A Magnetic Material Composed of Randomly Oriented SRRs," PIERS 2007, Beijing, China, CD-Rom, pp. 474-477, March 2007.

[3] J. Cajka, J. Kvasil, "Theory of Linear Circuits," SNTL, Prague 1979, p. 327, (in Czech). 